Resonance transition 795-nm rubidium laser using He buffer

gas

S. S. Wu, T. F. Soules, R. H. Page, S. C. Mitchell, V. K. Kanz, R. J. Beach

May 9, 2008

High-Power Laser Ablation 2008

Taos, NM, United States

April 20, 2008 through April 25, 2008 
This document was prepared as an account of work sponsored by an agency of the United States government. Neither the United States government nor Lawrence Livermore National Security, LLC, nor any of their employees makes any warranty, expressed or implied, or assumes any legal liability or responsibility for the accuracy, completeness, or usefulness of any information, apparatus, product, or process disclosed, or represents that its use would not infringe privately owned rights. Reference herein to any specific commercial product, process, or service by trade name, trademark, manufacturer, or otherwise does not necessarily constitute or imply its endorsement, recommendation, or favoring by the United States government or Lawrence Livermore National Security, LLC. The views and opinions of authors expressed herein do not necessarily state or reflect those of the United States government or Lawrence Livermore National Security, LLC, and shall not be used for advertising or product endorsement purposes. 


\title{
Resonance transition 795-nm rubidium laser using He buffer gas
}

\author{
Sheldon S.Q. Wu, ${ }^{\mathrm{a}, \mathrm{b}}$ Thomas F. Soules, ${ }^{\mathrm{a}}$ Ralph H. Page, ${ }^{\mathrm{a}}$ Scott C. Mitchell, ${ }^{\mathrm{a}}$ V. Keith Kanz, ${ }^{\mathrm{a}}$ \\ Raymond J. Beach ${ }^{\mathrm{a}}$ \\ ${ }^{a}$ Lawrence Livermore National Laboratory, 7000 East Avenue, Livermore, California 94551 \\ ${ }^{b}$ Department of Electrical and Computer Engineering, University of California at San Diego, La \\ Jolla, California 92093-0407
}

\begin{abstract}
Resonance transition rubidium laser $\left(5^{2} \mathrm{P}_{1 / 2} \rightarrow 5^{2} \mathrm{~S}_{1 / 2}\right)$ is demonstrated with a hydrocarbon-free buffer gas. Prior demonstrations of alkali resonance transition lasers have used ethane as either the buffer gas or a buffer gas component to promote rapid fine-structure mixing. However, our experience suggests that the alkali vapor reacts with the ethane producing carbon as one of the reaction products. This degrades long term laser reliability. Our recent experimental results with a "clean" helium-only buffer gas system pumped by a Ti:sapphire laser demonstrate all the advantages of the original alkali laser system, but without the reliability issues associated with the use of ethane. We further report a demonstration of a rubidium laser using a buffer gas consisting of pure ${ }^{3} \mathrm{He}$. Using isotopically enriched ${ }^{3} \mathrm{He}$ gas yields enhanced mixing of the $\mathrm{Rb}$ fine-structure levels. This enables efficient lasing at reduced $\mathrm{He}$ buffer gas pressure, improved thermal management in high average power $\mathrm{Rb}$ lasers and enhanced power scaling potential of such systems.
\end{abstract}

Keywords: rubidium laser, diode pumped alkali laser, DPAL

\section{INTRODUCTION}

Since the advent of lasers over four decades ago, solid-state and gas lasers have followed largely separate development paths with gas lasers being based primarily on direct electrical discharge pumping or luminescent chemical reactions, and dielectric solid-state lasers being pumped by flash lamps or semiconductor diode laser arrays. In 2002 researchers at Lawrence Livermore National Laboratory demonstrated a new class of laser, combining features from both the gas and solid state laser families, based on diode excitation of atomic alkali vapors. The first experimental demonstration of an optically pumped alkali laser in which $\mathrm{Rb}$ vapor in a buffer gas mixture consisting of $\sim 70$ Torr of ethane and $\sim 500$ Torr of He was lased under pump excitation from a Ti:sapphire laser [1]. Since then, several

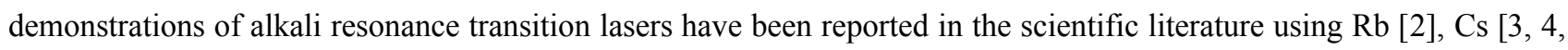
5] and $\mathrm{K}$ [6] as the gain media. A notable mention is the work done by Zhdanov et al which used extremely linenarrowed diode arrays, $\Delta \lambda<10 \mathrm{GHz}$, and demonstrated a $17 \mathrm{~W}$ laser with optical-optical efficiencies of $46 \%$ in $\mathrm{Rb}$ [7]. The concept of an optically pumped alkali resonance laser was proposed by Konefal in 1999 [8]. Konefal's proposal was for a longitudinally pumped alkali metal-molecular gas amplifier and was based on his experimental studies of collisionally induced fine-structure mixing in the presence of a molecular buffer gas such as ethane. Krupke extended Konefal's laser proposal to include diode-pumping by introducing a buffer gas component such as He that would broaden the $\mathrm{D}_{2}$ transition making it more accommodating of diode pump array linewidths. Because they are compatible with commercially available laser diode pump sources and show promise for power scaling with good output beam quality, diode-pumped alkali lasers (DPAL) are being actively investigated today. Most reported demonstrations done to date have used ethane as the buffer gas or a buffer gas component in the alkali vapor cell. This approach uses ethane to promote rapid fine-structure mixing, a requirement for efficient laser operation, between the terminal pump level $\left({ }^{2} \mathrm{P}_{3 / 2}\right)$ and the initial laser level $\left({ }^{2} \mathrm{P}_{1 / 2}\right)$. One issue with this approach is the chemical reaction that takes place between the alkali and the ethane. Although the deposition of carbon can be a slow process and may not impact experiments over hours of 
run time, the problem developed in all of our ethane cells eventually, especially higher temperature cells $\left(\mathrm{T}>135^{\circ} \mathrm{C}\right)$ as reported by Page et al [2]. Here we report experimental demonstration of the first Rb optical resonance transition lasers using pure He buffer gas. Our experimental results are compared with model predictions and support the use of a clean, hydrocarbon-free $\mathrm{Rb}-\mathrm{He}$ system for power scaling.

$\mathrm{Rb}$ has two dominant optical transitions, commonly referred to as the $\mathrm{D}_{1}$ and $\mathrm{D}_{2}$ lines. The two lines are located at 795 and $780 \mathrm{~nm}$ respectively and can be easily accessed by a variety of light sources including commercially available laser diode arrays. Optical gain is achieved by pumping on the $\mathrm{D}_{2}\left(5^{2} \mathrm{~S}_{1 / 2} \rightarrow 5^{2} \mathrm{P}_{3 / 2}\right)$ transition and extracting on the $\mathrm{D}_{1}$ $\left(5^{2} \mathrm{P}_{1 / 2} \rightarrow 5^{2} \mathrm{~S}_{1 / 2}\right)$ transition. While the $\mathrm{D}_{1}$ and $\mathrm{D}_{2}$ transitions are electric dipole transitions and possess large emission cross sections, the $5^{2} \mathrm{P}_{3 / 2} \rightarrow 5^{2} \mathrm{P}_{1 / 2}$ transition is not. Inelastic collisions with buffer gas atoms can provide the population transfer needed to reach inversion. Hence, to facilitate fast transfer of population between the fine-structure levels, a buffer gas is commonly added to the system. Fig 1 shows the Rb energy levels of interest.

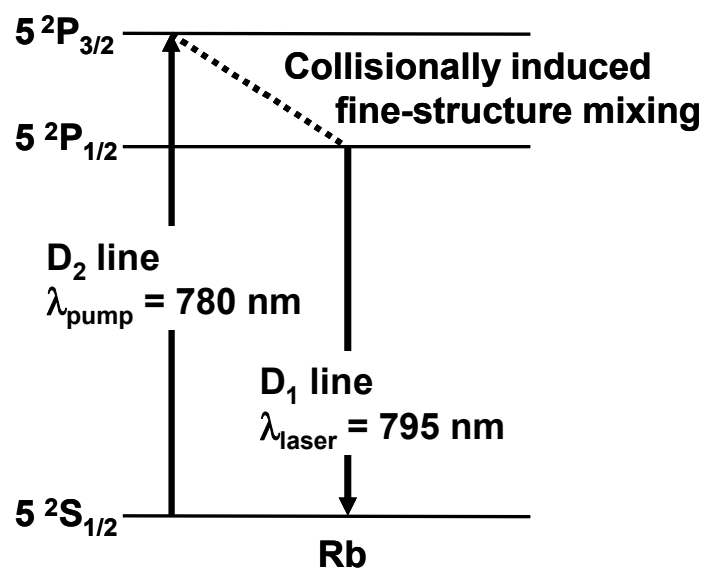

Fig. 1. Rb energy level diagram. Optical gain is achieved by pumping on the $\mathrm{D}_{2}$ transition and extracting on the $\mathrm{D}_{1}$ transition.

Ethane possesses a large $\mathrm{Rb}$ fine-structure mixing cross section and has been used in most reported demonstrations to effectively mix the upper states. However, one issue with this approach is the chemical reaction that takes place between the alkali and the ethane. The chemical reaction, $6 \mathrm{X}+\mathrm{C}_{2} \mathrm{H}_{6} \rightarrow 6 \mathrm{XH}+2 \mathrm{C}$ (graphite), where $\mathrm{X}$ is the alkali $(\mathrm{K}, \mathrm{Rb}$, or $\mathrm{Cs})$ and $\mathrm{XH}$ is the corresponding hydride, is thermodynamically favored and has a large negative free energy [9]. In our previous experiments that had used ethane as a component of the buffer gas, we observed that carbonaceous deposits formed at cell surfaces (e.g. windows) that simultaneously saw high intensity pump light and were exposed to alkali vapor and ethane. This negative effect was observed in numerous other reported demonstrations as well, e.g. [7]. Because we are ultimately interested in using end-pump geometries in which high intensity pump light is ducted through a cell via reflections with the side walls of the cell where pump light, ethane and alkali vapor meet together, it is essential that we mitigate this problem for reliable power scaling. While the predicted optical to optical efficiencies of $>60 \%$ have been demonstrated by workers in the field, confirming the anticipated scalability of alkali lasers, the issue of cell degradation has not been resolved. Helium, a gas commonly used to broaden the alkali transition lines, has been shown to possess some of the desirable properties of ethane but without the highly undesirable reactions. Beach et al calculated that the Rb-He fine-structure mixing cross section is sufficient to permit efficient diode-pumped $\mathrm{Rb}$ based systems at He buffer gas pressures of $\sim 10 \mathrm{~atm}$ and higher [3]. Obviated is the issue of carbon formation and degradation of the vapor cell that we observed in our previous alkali laser demonstrations that used ethane as a buffer gas component to promote rapid fine-structure mixing. 
One potential disadvantage of the pure He buffer gas approach arises from the smaller F-S mixing cross section of $\mathrm{Rb}-\mathrm{He}$, known to be orders of magnitude smaller than to that of Rb-ethane. The impact of these differing cross section values means that to achieve equivalent F-S mixing rates, higher pressures are required in the pure He systems than would be required in systems using ethane as a buffer gas component. However, higher He pressures lead to larger thermal aberrations under equivalent heat loads. The refractive index variation with temperature $\mathrm{dn} / \mathrm{dT}$ in the alkali gain cell is proportional to the He pressure, therefore higher He pressures will give larger thermal aberrations under equivalent heat loads. One approach to lowering the required He pressure in the alkali vapor gain cell is replacing the He buffer gas having a natural isotopic abundance with isotopically enriched ${ }^{3} \mathrm{He}$ (natural abundance of ${ }^{3} \mathrm{He}:{ }^{4} \mathrm{He}$ is approximately 1:740000).

The advantage of using isotopically enriched ${ }^{3} \mathrm{He}$ stems from its lower mass and therefore higher thermal velocity at a given temperature in comparison with naturally occurring He. The higher thermal velocity associated with ${ }^{3} \mathrm{He}$ increases the F-S mixing rate, $\gamma_{{ }^{2} P_{3 / 2} \rightarrow{ }^{2} P_{1 / 2}}=n_{H e} \sigma_{{ }^{2} P_{3 / 2} \rightarrow{ }^{2} P_{1 / 2}} v_{r}$, where $n_{H e}$ is the number of He atoms per unit volume, $\sigma_{{ }^{2} P_{3 / 2} \rightarrow{ }^{2} P_{1 / 2}}$ is the Rb-He F-S mixing cross section, and $v_{r}$ is the mean relative speed between $\mathrm{He}$ and $\mathrm{Rb}$ atoms. First, at a given temperature $v_{r}$ is higher in ${ }^{3} \mathrm{He}$ than ${ }^{4} \mathrm{He}$ by approximately $\sqrt{4 / 3} \approx 1.15$, which not only benefits the $\mathrm{F}$ $\mathrm{S}$ mixing rate which depends directly on $v_{r}$, but also improves thermal management in the cell. Since the thermal conductivity $\kappa$ of a gas to lowest order is proportional to the mean particle velocity, $\kappa$ of ${ }^{3} \mathrm{He}$ is larger than that of ${ }^{4} \mathrm{He}$ by the same factor. Secondly, the F-S mixing cross section itself has a velocity dependence that is expected to give a Rb${ }^{3} \mathrm{He}$ value larger than the $\mathrm{Rb}-{ }^{4} \mathrm{He}$ value at a given cell temperature due to the difference in thermal speeds of the two $\mathrm{He}$ isotopes [10]. It can be extrapolated from that velocity dependence that the Rb F-S mixing cross section in ${ }^{3} \mathrm{He}$ is approximately 1.5 times larger than that of ${ }^{4} \mathrm{He}$ at our operating temperatures. Together, one can expect a $\mathrm{Rb}$ F-S mixing rate about 1.7 times larger for the ${ }^{3} \mathrm{He}$ system than the ${ }^{4} \mathrm{He}$ system under same operating conditions.

Laser modeling was conducted following the formalism outlined in Ref [3] and summarized below. The model follows from a master equation approach in the rate equation approximation. Longitudinal averaging is used to simplify calculations without loss of applicability to high gain laser media. In the equations, $\mathrm{n}_{1}$ represents the population in the ground state $\left(5^{2} \mathrm{~S}_{1 / 2}\right)$, and $\mathrm{n}_{2}$ and $\mathrm{n}_{3}$ represent the populations in $5^{2} \mathrm{P}_{1 / 2}$ and $5^{2} \mathrm{P}_{3 / 2}$ states respectively. Collision induced excitation transfer rate between the fine-structure levels is represented by $\gamma$ 's. Quenching collision transfer rates to the ground state are negligibly small in the case of He buffer gas and need not to be considered in these calculations. The equations that govern the distribution of populations in the laser are

$$
\begin{aligned}
& \frac{d n_{3}}{d t}=\Gamma_{P}-\gamma_{{ }_{P_{3 / 2}} \rightarrow{ }^{2} P_{1 / 2}} n_{3}+\gamma_{{ }^{2} P_{1 / 2} \rightarrow{ }^{2} P_{3 / 2}} n_{2}-\frac{n_{3}}{\tau_{D_{2}}} \\
& \frac{d n_{2}}{d t}=-\Gamma_{L}+\gamma_{{ }^{2} P_{3 / 2} \rightarrow{ }^{2} P_{1 / 2}} n_{3}-\gamma_{{ }_{P_{1 / 2} \rightarrow{ }^{2} P_{3 / 2}}} n_{2}-\frac{n_{2}}{\tau_{D_{1}}} \\
& \frac{d n_{1}}{d t}=-\Gamma_{P}+\Gamma_{L}+\frac{n_{2}}{\tau_{D_{1}}}+\frac{n_{3}}{\tau_{D_{2}}}
\end{aligned}
$$

where $\Gamma_{\mathrm{P}}$ and $\Gamma_{\mathrm{L}}$ are the transition rates associated with pump photon absorption and laser photon emission respectively. We do not assume that local thermal equilibrium exists a priori between the $5^{2} \mathrm{P}_{1 / 2}$ and the $5^{2} \mathrm{P}_{3 / 2}$ levels because of the limited $\mathrm{Rb}-\mathrm{He}$ fine structure mixing rate and continue to carry the full set of rate equations in our modeling of the alkali system. The appropriate expressions for $\Gamma_{\mathrm{P}}$ and $\Gamma_{\mathrm{L}}$ are 


$$
\begin{aligned}
& \Gamma_{P}=\frac{\eta_{\text {mode }} \eta_{\text {del }}}{V_{L}} \int d \lambda \frac{P_{\text {pump }}(\lambda)}{h c / \lambda}\left[1-e^{-\left(n_{1}-\frac{1}{2} n_{3}\right) \sigma_{D_{2}}(\lambda) l}\right]\left[1+R_{P} e^{-\left(n_{1}-\frac{1}{2} n_{3}\right) \sigma_{D_{2}}(\lambda) l}\right] \\
& \Gamma_{L}=\frac{1}{V_{L}} \frac{P_{\text {laser }}}{h c / \lambda_{D 1}} \frac{R_{O C}}{1-R_{O C}} T_{\text {window }}\left(\frac{1}{T_{\text {window }}^{2} \sqrt{R_{O C} R_{H R}}}-1\right)\left(1+\sqrt{\frac{R_{H R}}{R_{O C}}}\right)
\end{aligned}
$$

where $\mathrm{V}_{\mathrm{L}}$ is the volume of the laser mode, $\eta_{\text {mode }}$ is the fraction of the pump light that intercepts the laser mode's crosssectional area and thus contributes to lasing, $\eta_{\text {del }}$ is the fraction of the pump power delivered from the pump excitation source to the input of the laser gain medium, $\mathrm{P}_{\text {pump }}(\lambda)$ is the spectrally resolved pump power, $\mathrm{R}_{\mathrm{P}}$ represents the reflectivity of the pump light on the high reflecting mirror after single passing the laser gain medium, $\sigma_{\mathrm{D} 2}(\lambda)$ is the spectrally resolved pump absorption cross section, $T_{\text {window }}$ is the transmission efficiency through a single cell window. All mixing rates, lifetimes, broadening rates, and cross sections are taken from published scientific literature.

Based on our model predictions, the lack of ethane induced mixing of the Rb fine-structure levels can be sufficiently overcome by a higher density of He. Due to the small spectral linewidth of the pump source used for this demonstration, population inversion between the lasing levels can be attained at He pressures of a few atmospheres. We project that in diode-pumped power scaled systems, optical to optical efficiencies of $>70 \%$ can be reached with the same hydrocarbon-free approach using conventional diode arrays. The use of atmospheres of $\mathrm{He}$ as the buffer gas is compatible with pump linewidths of $\sim 0.5 \mathrm{~nm}$, a regime requiring only modest linewidth control with today's conventional 2-d stacks of laser diode array technology. Hydrocarbon-free diode-pumped alkali lasers present a new pathway to high average power with good beam quality and high efficiency.

\section{EXPERIMENT}

A schematic diagram for the experimental setup used in this demonstrate of hydrocarbon-free $\mathrm{Rb}$ laser is shown in Fig. 2. The Rb vapor and He gas were contained in a $3 \mathrm{~cm}$ long cylindrical ceramic cell with sapphire windows that were AR coated on their external surfaces, but uncoated on their internal surfaces. Rb was introduced into the cell as a pure metal. This was followed by cell evacuation using a laboratory vacuum system and then the introduction of $40 \mathrm{psi}$ of $\mathrm{He}$ gas $(2.7 \mathrm{~atm})$ at room temperature $\left(\sim 20^{\circ} \mathrm{C}\right)$. The cell was placed in a close-fit copper oven with electric heaters that allowed us to maintain the cell temperature to within $1{ }^{\circ} \mathrm{C}$ and to fix the resulting $\mathrm{Rb}$ saturated vapor pressure inside

the cell. The entire cell was heated to temperatures up to $170^{\circ} \mathrm{C}$, corresponding to a $\mathrm{Rb}$ vapor density of $2.2 \times 10^{14} \mathrm{~cm}^{-3}$ inside the cell. 


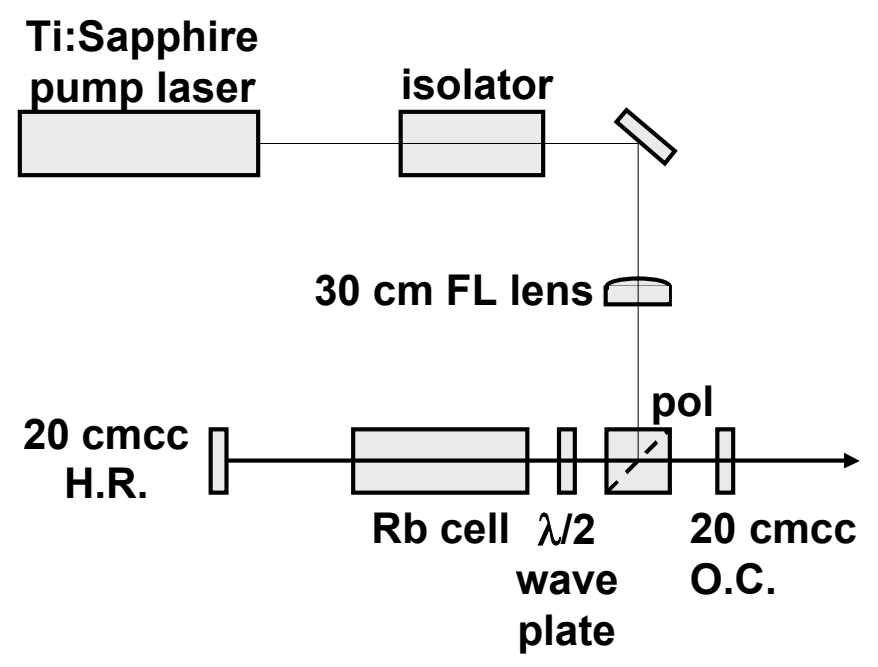

Fig. 2. Schematic diagram of the experimental setup used in our demonstrations. The laser cavity mirrors have $\sim 20 \mathrm{~cm}$ radii of curvature and are both concave (cc). H.R. stands for high reflector, O.C. for output coupler and FL for focal length.

The pump source used was a Ti:sapphire laser that produced up to $2.7 \mathrm{~W}$ of linearly polarized, near-diffractionlimited CW optical radiation. The Ti:sapphire laser linewidth was $\sim 9 \mathrm{GHz}$ FWHM making the pump laser source narrow compared to the He-broadened $\mathrm{D}_{2}$ pump absorption feature, which we estimate is $\sim 50 \mathrm{GHz}$ wide based on the known $\mathrm{Rb}-\mathrm{He}$ collisional broadening rate of $18.1 \mathrm{GHz} /$ amagat [11]. The pump light was coupled into the $40.5 \mathrm{~cm}$ long laser cavity via a polarizing beam splitter and traversed the vapor cell twice by reflecting off the highly reflecting end mirror. The end mirror has 0.99 reflectivity at both the pump and lasing wavelengths. The pump beam was aligned parallel to the laser cavity axis and focused to a $220 \mu \mathrm{m}$ diameter spot size at the center of the cell, resulting in peak pump irradiances of nearly $5 \mathrm{~kW} / \mathrm{cm}^{2}$. Since the optic axes of the pair of sapphire windows were set at unknown orientations, a half-wave plate was placed in the cavity to partially compensate for the polarization changes caused by birefringence. We estimate that even with the half-wave plate, laser light traversing the cell had a $75 \%$ transmission efficiency passing the cube polarizer on its return path.

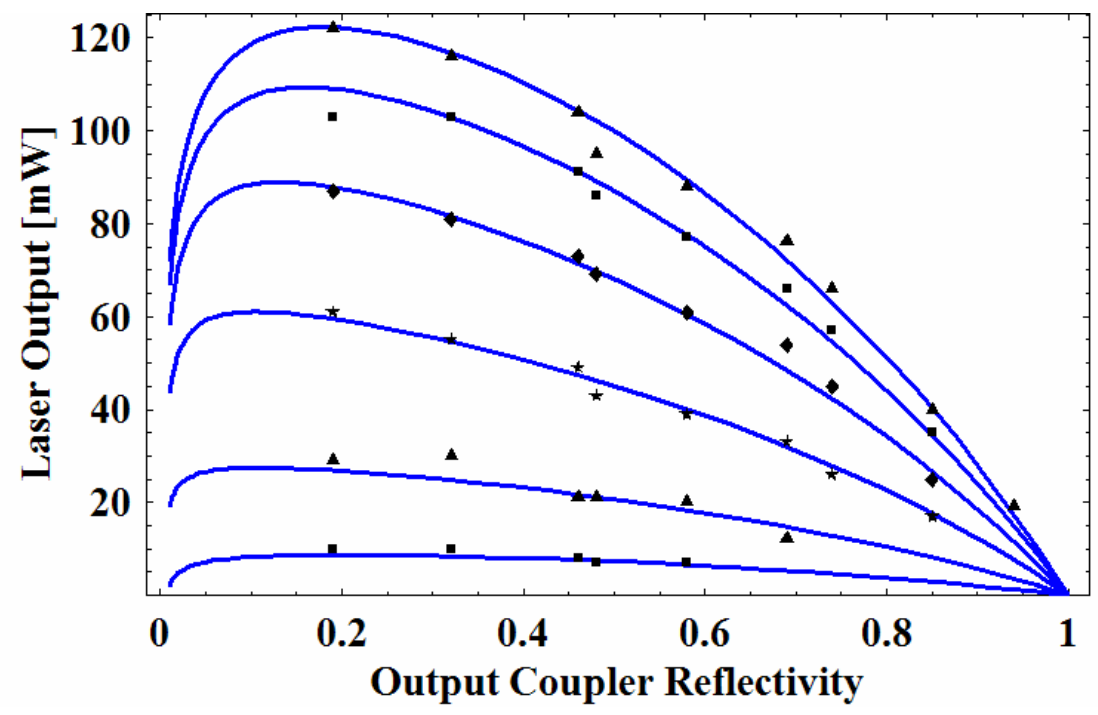

Fig. 3. Rb laser output power for various pump powers plotted against output coupler reflectivity. Solid curves represent model predictions. From top to bottom, pump powers are: $1.81 \mathrm{~W}, 1.53 \mathrm{~W}, 1.23 \mathrm{~W}, 0.93 \mathrm{~W}, 0.63 \mathrm{~W}, 0.47 \mathrm{~W}$. 
We observed linearly polarized $\mathrm{CW}$ laser emission at $795 \mathrm{~nm}$ in a $\mathrm{TEM}_{00}$ beam at cell temperatures in the vicinity of $145^{\circ} \mathrm{C}$. Maximum powers of over $130 \mathrm{~mW}$ and optical to optical efficiencies close to $7 \%$ were measured despite single-pass passive optical losses near $40 \%$ in our laser cavity from cube polarizer and window reflections. Presented in Fig. 3 is the theoretical and measured laser output power versus output coupler reflectivity at several Ti:sapphire pump laser powers. The reported pump powers were measured outside the laser cavity and delivered into the laser cavity with an efficiency close to 0.9. Large cavity losses along with the high gains that characterize alkali atoms put the optimal output coupler reflectivity at below 0.2 . These high values for optimized output coupling support the use of geometrically unstable resonators for the scaling of diode-pumped alkali lasers to high power with good beam quality. The theoretical curves overlaying the experimental data points were calculated using our laser model. Using experimentally measured values and treating the beam overlap and the $\mathrm{Rb}-\mathrm{He}$ fine-structure mixing rate as adjustable parameters, we were able to find excellent agreement between our model and laser output data. The model curves were generated using an effective $\mathrm{Rb}$-He fine-structure mixing cross section value of $4.6 \times 10^{-17} \mathrm{~cm}^{2}$ and $24 \%$ mode overlap efficiency. Mode overlap is defined as in [3] as the fraction of the pump excited volume in the alkali cell extracted by the circulating laser radiation in the resonator. We attribute the low overlap efficiency to a mismatch between the pump and laser beam waists at the cell center, the pump laser beam waist (1/e-HW) being measured to be $110 \mu \mathrm{m}$ and the laser mode beam waist estimated to be $75 \mu \mathrm{m}$ at cell center, and the imperfect collinear overlap of the pump and the laser beams through the length of the $3 \mathrm{~cm}$ long cell. We note the Rb-He fine-structure mixing rate that gave the best fit between our model and experimental data is approximately three times larger than that predicted based on cross sections previously reported by Gallagher [10]. Fig. 4 shows a plot of measured laser output power against cell temperature, acquired as the cell temperature was continuously varied between 115 and $160^{\circ} \mathrm{C}$. For this data the output coupler had a measured reflectivity of 0.19 . The model parameter values used to generate the model overlay of the data in Fig. 4 are the same as those used for the overlay shown in Fig. 3.

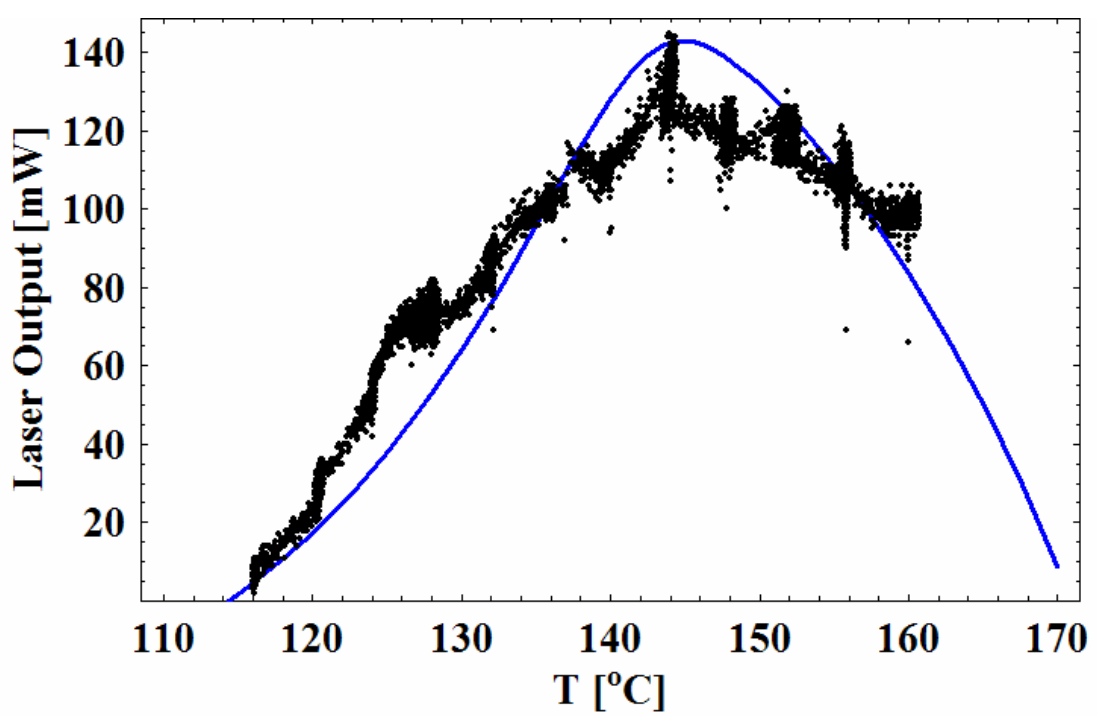

Fig. 4. Rb laser output power with varying cell temperature using a 0.19 reflectivity output coupler. Solid curve shows model prediction.

Fig. 5 is a different plot of the data from Fig. 3 illustrating a feature of the Rb laser using pure He buffer gas. Both our data and the overlaid laser model predict a decrease or roll off in slope efficiency at higher pump powers. This decrease in slope efficiency is caused by the limited mixing rates between the Rb fine-structure energy levels. After a $\mathrm{Rb}$ atom is optically excited to $5^{2} \mathrm{P}_{3 / 2}$ energy level, it radiatively returns to the $5^{2} \mathrm{~S}_{1 / 2}$ ground level or transfers its energy via He collision to the $5^{2} \mathrm{P}_{1 / 2}$ initial laser level. Under strong pumping, the limited fine-structure mixing rate is 
insufficient in keeping the initial laser level appropriately filled for optimum laser operation, i.e. the initial laser level is essentially starved for population at the higher pump excitation rates resulting in the observed roll off in slope efficiency. For this demonstration of the hydrocarbon-free rubidium laser, we purposely picked operating conditions suitable for the Ti:sapphire pump laser that also present a slight bottleneck in laser performance. Agreement between model and experiment supports our understanding of the underlying physical processes. Note that this saturation behavior is expected to be negligible for power scaled diode pumped systems which operate at significantly higher He pressures.

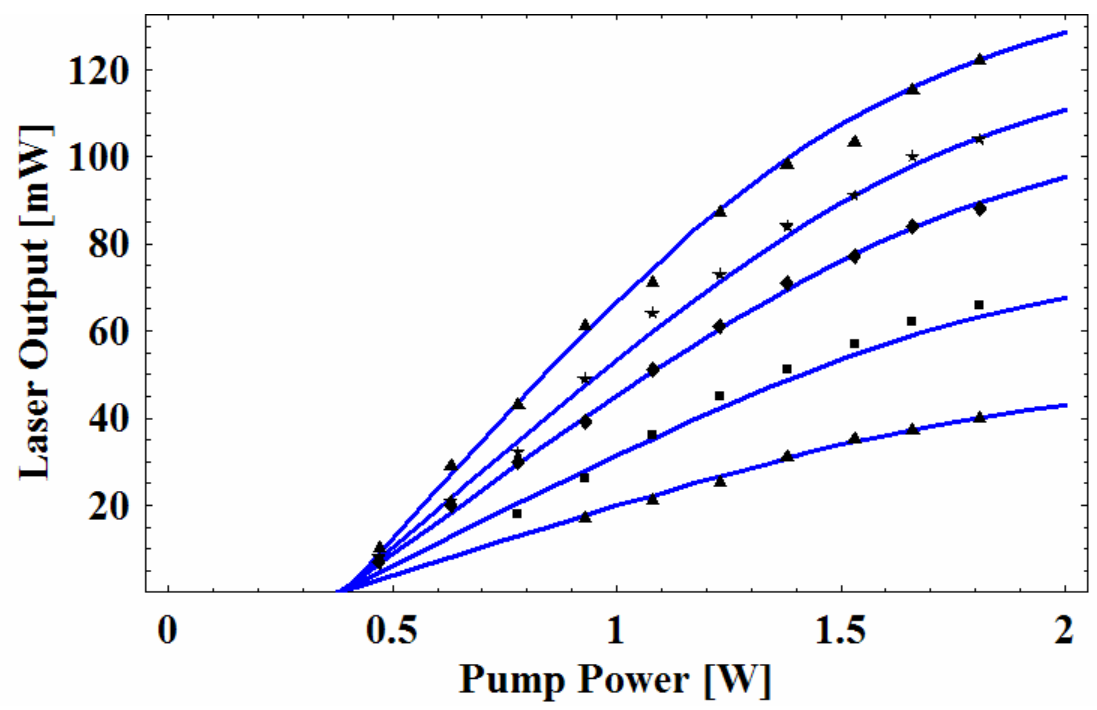

Fig. 5. $\mathrm{Rb}$ laser output power for various output couplers plotted against pump power. Solid curves represent model predictions. From top to bottom, the reflectivities are: $0.19,0.46,0.58,0.74,0.85$. The reflectivities were measured using a Ti:sapphire probe beam at $795 \mathrm{~nm}$.

We have in addition demonstrated the operation of a 795-nm Rubidium resonance laser system using a buffer gas consisting of pure ${ }^{3} \mathrm{He}$. The experimental setup used in this $\mathrm{Rb}-{ }^{3} \mathrm{He}$ demonstration is nearly identical to that shown in Fig 2. The ${ }^{3} \mathrm{He}$ gas is manufactured by Spectra Gases Inc. with a quoted $99.999 \%$ chemical purity and $99.9 \%$ isotopic enrichment. Laser emission at $795 \mathrm{~nm}$ in a $\mathrm{TEM}_{00}$ beam was observed at cell temperatures in the vicinity of $150^{\circ} \mathrm{C}$. Maximum output powers of over $350 \mathrm{~mW}$ were measured, corresponding to an optical-optical efficiency greater than 21\%. Presented in Figure 6 is the theoretical and measured laser output power versus pump power using different output couplers at a cell temperature of $142^{\circ} \mathrm{C}$. Because of the larger $\mathrm{Rb}^{3}{ }^{3} \mathrm{He}$ inelastic collision cross section compared to that of ${ }^{4} \mathrm{He}$, the mixing rate between the F-S levels is large enough to maintain efficient lasing at these pump powers. The saturation effect observed in our previous demonstration for the $\mathrm{Rb}-{ }^{4} \mathrm{He}$ laser in which the slope efficiency decreased at higher pump powers due to insufficient F-S mixing is significantly diminished here with nearly identical operating conditions, supporting the use of ${ }^{3} \mathrm{He}$ with its larger F-S mixing rate for power scaled systems. Based on measured pump beam characteristics and the cavity dimensions, we treated the $\mathrm{Rb}-{ }^{3} \mathrm{He}$ F-S mixing rate and the mode overlap efficiency as adjustable parameters over a reasonable range. The model curves were generated using an effective $\mathrm{Rb}-$ ${ }^{3} \mathrm{He}$ F-S mixing cross section value of $7.1 \times 10^{-17} \mathrm{~cm}^{2}$ and $57 \%$ mode overlap efficiency. The larger mode overlap efficiency is a major source of the increase in laser output power compared with our $\mathrm{Rb}-{ }^{4} \mathrm{He}$ laser results. The $\mathrm{Rb}-{ }^{3} \mathrm{He}$ fine-structure mixing rate that gives the best fit between our model and experimental data is, as expected, approximately 1.5 times larger than our model-fitted value for the $\mathrm{Rb}^{-}{ }^{4} \mathrm{He}$ cross section. The higher F-S mixing rate, which is critical for efficient laser performance, enables comparable performing ${ }^{3} \mathrm{He}$ systems at only $\sim 60 \%$ of the He buffer gas pressure required for the ${ }^{4} \mathrm{He}$ systems. Since thermal aberrations in the laser's $\mathrm{Rb}$ vapor cell are governed by $\mathrm{dn} / \mathrm{dT}$ which is proportional to the He pressure, the ${ }^{3} \mathrm{He}$ approach will be advantageous for high beam quality lasers in power scaled 
systems [3]. Another benefit of ${ }^{3} \mathrm{He}$ based systems over ${ }^{4} \mathrm{He}$ based systems is the higher thermal conductivity $\kappa$ of ${ }^{3} \mathrm{He}$, $\kappa_{{ }^{3} \mathrm{He}}: \kappa_{{ }^{4} \mathrm{He}} \sim 1.15: 1$ - an important consideration in thermal management driven designs of power scaled systems.

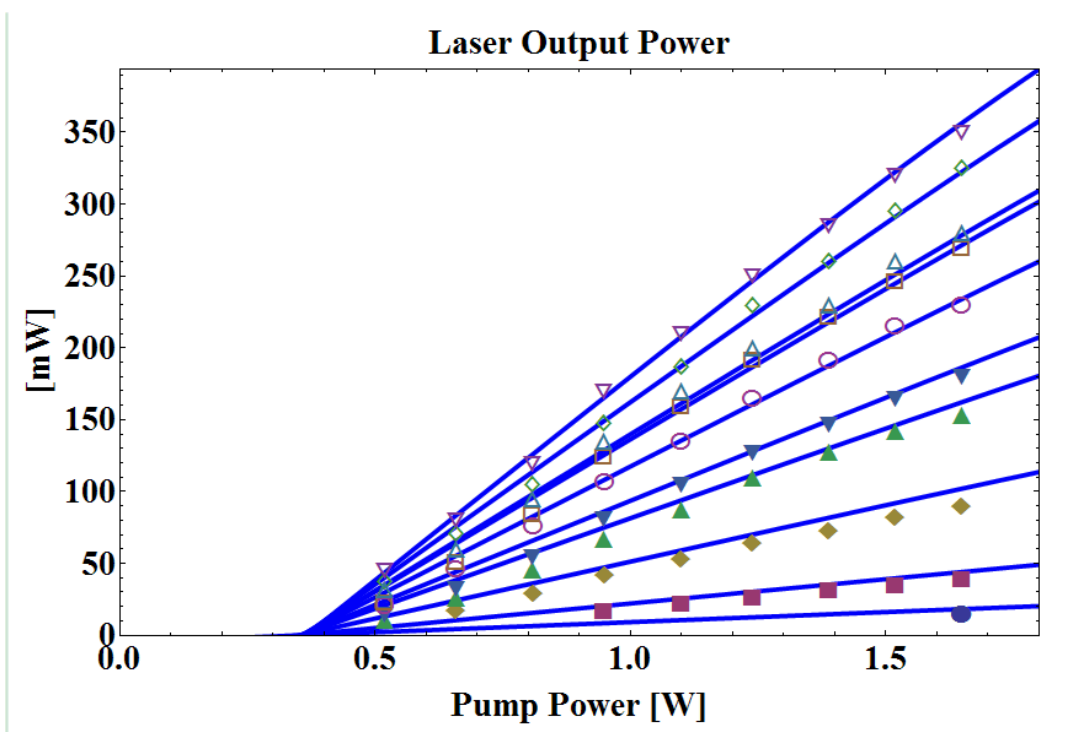

Fig. 6. Rb laser output power for various output couplers plotted against pump power. Solid curves represent model predictions. From top to bottom, the reflectivities are: $0.19,0.32,0.46,0.48,0.58,0.69,0.74,0.85,0.94$ and 0.976 . The reflectivities were measured using a Ti:sapphire probe beam at $795 \mathrm{~nm}$.

The same clean, hydrocarbon-free approach being reported here for $\mathrm{Rb}$ based systems is also applicable for $\mathrm{K}$ based systems, but not Cs-based systems. This is because the Cs-He fine-structure mixing cross section is too small to make a He-only buffer gas system feasible using Cs. The reported value for the Cs-He fine structure mixing cross section is almost three orders of magnitude smaller than the Rb-He cross section value [10].

\section{CONCLUSIONS}

In summary, two realizations of hydrocarbon-free optical resonance transition rubidium laser have been demonstrated. Good agreement between measured laser performance and our laser model supports further pursuing this approach for power scaling. Obviated is the issue of carbon formation and degradation of the vapor cell that we observed in our previous alkali laser demonstrations that used ethane as a buffer gas component to promote rapid finestructure mixing. We have been able to reuse the cell for multiple heating cycles and helium fills without any signs of degradation. The use of isotopically enriched ${ }^{3} \mathrm{He}$ offers multiple advantages over the use of naturally occurring He. The higher thermal velocity of ${ }^{3} \mathrm{He}$ over that of ${ }^{4} \mathrm{He}$ give a Rb F-S mixing rate about 1.7 times larger for the ${ }^{3} \mathrm{He}$ system than the ${ }^{4} \mathrm{He}$ system under the same operating conditions. The higher F-S mixing rate is critical for efficient laser performance and eases thermal management requirements. In view of these advantages of ${ }^{3} \mathrm{He}$ over ${ }^{4} \mathrm{He}$ based systems, we expect the ${ }^{3} \mathrm{He}$ approach to be the preferred route to power scaling DPAL lasers to efficient, reliable, and good beam quality systems. The use of atmospheres of He as the buffer gas is compatible with pump linewidths of $\sim 0.5 \mathrm{~nm}$, a regime requiring only modest linewidth control with today's conventional 2-d stacks of laser diode array technology. Because of its model projected efficiency advantages over diode-pumped solid state lasers (DPSSLs), its compatibility with commercially available laser diode arrays, and now a demonstrated system that promise very high reliability, diodepumped He-only $\mathrm{Rb}$ lasers present a new pathway to high average power with good beam quality and high efficiency. 
We are grateful to Prof. Paul Yu of UC San Diego; John O’Pray, Mike Tobin, Kevin Zondervan, Jim Kotora, Denise Podolski, Mark Rotter, all of the Missile Defense Agency, Jeff Thomas, Eliot Geathers, Tom Leheka, Brian Brickeen, Dave Bernot, all of the Penn State Electro Optics Center, and Chris Barty of Lawrence Livermore National Laboratory for their support and many useful discussions. We also gratefully acknowledge the financial support provided by the Missile Defense Agency and the Directed Energy Professional Society. This work performed under the auspices of the U.S. Department of Energy by Lawrence Livermore National Laboratory under Contract DE-AC5207 NA27344.

\section{REFERENCES}

1 W. F. Krupke, R. J. Beach, V. K. Kanz, and S. A. Payne, "Resonance transition 795-nm rubidium laser," Opt. Lett. 28, 2336-2338 (2003).

2 R. H. Page, R. J. Beach, V. K. Kanz, and W. F. Krupke, "Multimode-diode-pumped gas (alkali-vapor) laser," Opt. Lett. 31, 353-355 (2006)

3 R. J. Beach, W. F. Krupke, V. K. Kanz, S. A. Payne, M. A. Dubinskii, and L. O. Merkle, "End-pumped continuouswave alkali vapor lasers: experiment, model, and power scaling," J. Opt. Soc. Am. B 21, 2151-2163 (2004).

4 T. Ehrenreich, B. Zhdanov, T. Takekoshi, S. P. Phipps, and R. J. Knize, ”Diode Pumped Cesium Laser", Electronics Lett. 41, 47-48 (2005).

5 Y. Wang, T. Kasamatsu, Y. Zheng, H. Miyajima, H. Fukuoka, S. Matsuoka, M.Niigaki, H. Kubomura, T. Hiruma, H. Kan, " Cesium vapor laser pumped by a volume-Bragg-grating coupled quasi-continuous-wave laser-diode array", Appl. Phys. Lett. 88, 141112 (2006).

${ }_{6}$ B. Zhdanov, C. Maes, T. Ehrenreich, A. Havko, N. Koval, T. Meeker, B. Worker, B. Flusche and R. J. Knize, “Optically Pumped Potassium Laser”, Opt. Com. 270, 353-355 (2007).

7 B. V. Zhdanov, A. Stooke, G. Boyadjian, A. Voci, and R. J. Knize, "Rubidium vapor laser pumped by two laser diode arrays," Opt. Lett. 33, 414-415 (2008)

8 Z. Konefal, "Observation of collision induced processes in rubidium-ethane vapour", Opt. Com. 164, 95-105 (1999).

9 FactSage 5.5 $5^{\mathrm{TM}} / \mathrm{FactWeb}^{\mathrm{TM}}, \mathrm{CRCT}$, Ecole Polytechnique, Montreal, Canada, (2007).

10 A. Gallagher, "Rubidium and Cesium Excitation Transfer in Nearly Adiabatic Collisions with Inert Gases", Phys. Rev. 172, 88 (1968).

11 M. V. Romalis, E. Miron, and G. D. Gates, "Pressure broadening of $\mathrm{Rb}^{\mathrm{D}_{1}}$ and $\mathrm{D}_{2}$ lines by ${ }^{3} \mathrm{He},{ }^{4} \mathrm{He}, \mathrm{N}_{2}$, and Xe: Line cores and near wings", Phys. Rev. A 56, 4569-4578 (1997). 